\title{
A Two-Dimensional Deflection Sensor Based on Force Sensing Resistors
}

\author{
Chuangqiang Guo, Chunya Wu, Bin Wang, and Hong Liu \\ State Key Laboratory of Robotics and System, Harbin Institute of Technology, Harbin 150001, China \\ Correspondence should be addressed to Chunya Wu; wuchunya1982@163.com
}

Received 10 March 2017; Revised 17 May 2017; Accepted 22 May 2017; Published 5 July 2017

Academic Editor: Lucio Pancheri

Copyright ( 2017 Chuangqiang Guo et al. This is an open access article distributed under the Creative Commons Attribution License, which permits unrestricted use, distribution, and reproduction in any medium, provided the original work is properly cited.

\begin{abstract}
A flexible deflection sensor for elastic shaft with the capability of measuring the amplitude and direction of bending is introduced in this paper. A thin force sensing resistors (FSR) film is taken as its basic material, which is sandwiched by an elastomer layer and a printed circuit board (PCB) with detecting electrode grids. Two fix rings are used to fix the three thin components perpendicularly to the longitudinal direction of the flexible shaft. When the shaft bends under forces, the fix rings will generate a normal pressure on FSR, which will cause the change of the resistance. Therefore, the amplitude of bending can be got based on the value of resistance. The electrode grid on the PCB is divided into four detection areas used to estimate the distribution of normal pressure on the FSR; thus the bending direction of shaft can also be obtained. Test results of a prototype (140 $\mathrm{mm}$ in length) show that the amplitude of deflection can reach $30 \mathrm{~mm}$ and the sensitivities of sensor are $40.37,32.8,37.77$, and $39.47 \mathrm{mV} / \mathrm{mm}$ in the four directions, respectively. The proposed flexible deflection sensor can be applied in continuum robots or other applications, which require rapid measurement of bending amplitude and direction.
\end{abstract}

\section{Introduction}

Control of a robot always involves solving the forward kinematics problem in real-time as a means to compute the desired twist (velocity) vector for the tip coordinate frame [1]. For the conventional robots comprised of rigid links and discrete joints, position or speed sensors are usually integrated at the joints for the kinematic calculation. Continuum robots, however, which obtain their shape through the flexure of structural components, often require more complex mechanics-based models [2]. Thus, a set of sensors positioned along the longitudinal direction of the continuum robot are always needed to measure the local curvature, from which the shape of robot can be computed in real-time.

Approaches to measuring the shape of a continuum robot include image-based methods [3, 4], electromagnetic tracking [5], force sensing in control tendons [6], and optical fibers with fiber Bragg gratings (FBGs) $[1,2]$. Despite the fact that each of these approaches can work well in a certain situation, they also have some shortcomings. For example, image-based methods usually require computing intensively, leading to low update rates [1]. Electromagnetic tracking is sensitive to magnetic field distortions and often only provides the location of robot tip rather than the shape of the entire robot. Tendon force sensing is corrupted by tendon sliding friction [1]. In reference [7, 8], a dynamic model of the tend-driven elongated surgical robotic manipulator and several impressive estimated methods for grip force, based on Unscented Kalman Filter (UKF) and Gaussian Process Regression (GPR), were proposed, to minimize the impact from the friction and other factors (cable stretch, movement, etc.). However, although these methods can be useful to estimate the terminal state of the instrument, the detailed information of the entire shape is still difficult to be provided. Furthermore, the shape information can be directly measured by optical fibers, but complex optical devices are needed to be introduced, which will bring other inconvenience [2].

Since the deflection sensor (DS) can measure the angular displacement between two points which lie on a common substrate [9], the entire shape of the robot can be recovered and monitored by measuring the displacement between a series of consecutive points. The displacement corresponds to the deformation of the substrate, so deflection may be reflected by the stress or strain on the material arising 
from the deformation [10]. For a strain sensor, which is expected to monitor a bending actuator without influencing the strain field, it needs to bend easily with a large working range (up to $10 \%$ strain) and be suitable for low frequencies $(<10 \mathrm{~Hz})[11]$. Thus, the traditional strain gauges limited by the elastic strain range are not fit for such kind of task, and some new ideas of deflection sensor were conceived. The polyvinylidene difluoride (PVDF) film has been adopted to measure the deflection of bi-bellows actuator; however, it can only bend at one direction without elongation [11]. Also, a new contactless deflection sensor was proposed using LED, but the application was still restricted to the measurement of deflection at one direction [9].

In this paper, we report a new flexible deflection sensor with the capability of measuring the bending scope and the direction of continuum robots, using the force sensing resistors (FSR) as a basic material. The sensor can be mounted outside of the shaft perpendicularly to the longitudinal direction. When the shaft bends under the internal or external forces, the normal pressure will be exerted on the FSR film along the bending direction. According to the resistance change of FSR film, the deflection and the bending direction of the shaft can be detected. In the following sections of this paper, the structure and the operation principle of the sensor will be analyzed in detail first. Then the prototype and the fabrication processes together with the method used to measure the bending direction will be introduced. The scanning method to eliminate the effect of the crosstalk will be presented. Finally, the experimental results of the developed prototype will be discussed.

\section{Materials and Methods}

2.1. Structure and Basic Operating Principle. The proposed deflection sensor, composed of 5 concentric rings, is installed on the outer side of a core shaft, as shown in Figure 1. The FSR layer with a thickness of $\sim 0.5 \mathrm{~mm}$ is located in the middle of the sensor to measure the deformation of the core shaft, according to the change of its own resistance under the external pressure. An elastic rubber layer with appropriate hardness and thickness is placed under the FSR film to endow the sensor with the suitable filtering capacity of external pressure, making the external force distributed uniformly on the FSR film. The other side of the FSR film is in direct contact with the electrode grids of a printed circuit board (PCB). The electrode grids are divided into power supply electrodes and measuring electrodes, which are alternatively distributed around the circuit board. The three layers are fixed on the shaft by two fix rings, which are also used to exert normal stress $\left(F_{n}\right)$ on the FSR layer when the core shaft bends under the force $(F)$. Under the combined action of the supply voltage and the external pressure, the change of FSR resistance will be indicated by the change of the electrode current accordingly. Therefore, the deflection of the shaft could be obtained by detecting the current output from the measuring electrodes on the circuit board. Furthermore, if a plurality of sensors are mounted along the longitudinal direction of the target robot, the shape of the entire robot body can be gained.

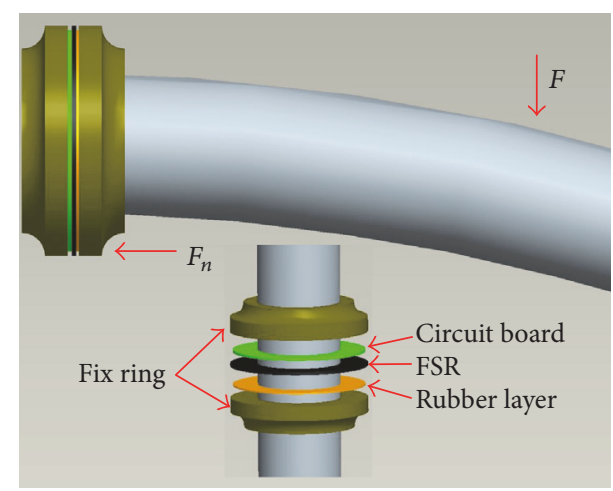

FIGURE 1: Conceptual diagram of the proposed deflection sensor.

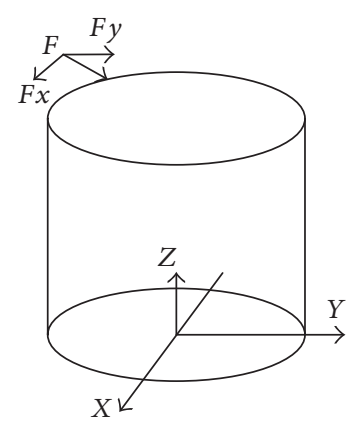

(a)

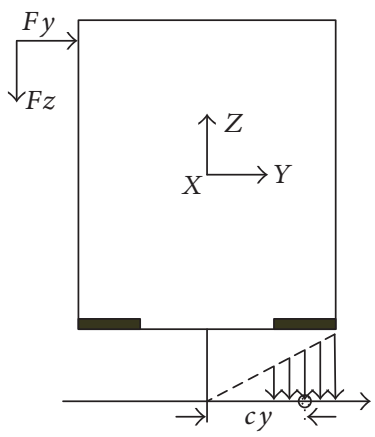

(c)

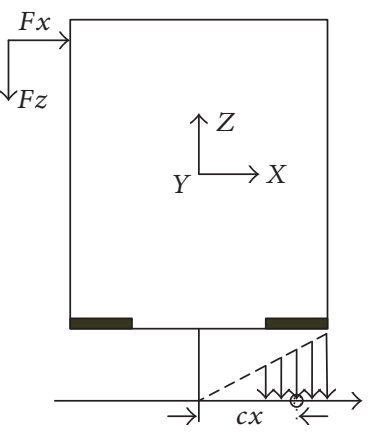

(b)

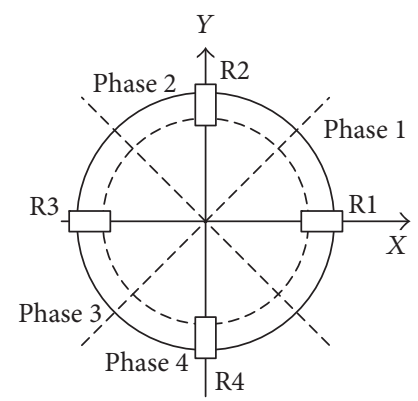

(d)
FIGURE 2: Mechanism of the proposed deflection sensor.

The basic mechanism of the proposed deflection sensor is that the stress distribution and the force on the FSR, which can be measured, are used to estimate the imposed external forces and the deflection of the core shaft. In order to measure the bending direction of the shaft, a specific coordinate system is assumed to describe the distribution of stress on the proposed sensor. As shown in Figure 2(a), the $X-Y$ coordinate plane is defined to be perpendicular to the center line and the $Z$-axis is set to coincide with the center line of the core shaft. The component of the force $F$ in $X$ direction (defined as $F_{x}$ ) will act on both sides of $X$ axis on the $X-Y$ plane. The central angle will be restricted within $(-\pi / 4, \pi / 4]$ or $(3 \pi / 4,5 \pi / 4]$, as shown in Figure $2(\mathrm{~b})$. Similarly, the component of the force in $Y$ direction (defined as $F_{y}$ ) will act on both sides of $Y$-axis on the $X-Y$ plane. The central angle will be restricted within $(\pi / 4,3 \pi / 4]$ or $(5 \pi / 4$, 


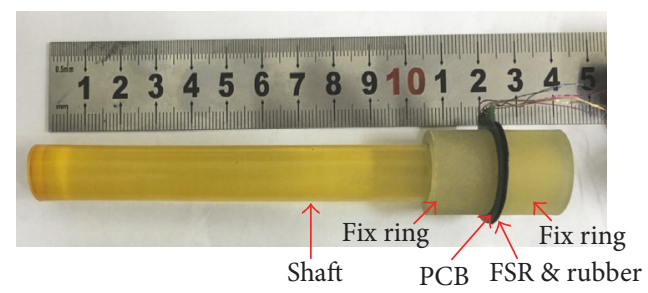

FIGURE 3: Prototype of the proposed sensor.

$7 \pi / 4]$, as shown in Figure 2(c). Therefore, the $X-Y$ plane can be divided into four measuring phases (phase 1 phase 4 ), as shown in Figure 2(d). The amplitude and direction of the action force $F$ and deflection can be gotten by measuring the resistors of FSR in each phase (R1 R4).

Regarding the installation position of the sensor, the core shaft can only bend in one direction at a given time, so the force $F_{x}$ (or $F_{y}$ ) cannot act on both the positive and the negative directions of $X$ (or $Y$ ) axis at one time. Therefore, the $F_{x}$ and $F_{y}$ can be defined by

$$
\begin{aligned}
& F_{x}= \begin{cases}f_{1}\left(V_{1}\right) & F_{x} \geq 0 \\
f_{3}\left(V_{3}\right) & F_{x}<0\end{cases} \\
& F_{y}= \begin{cases}f_{2}\left(V_{2}\right) & F_{y} \geq 0 \\
f_{4}\left(V_{4}\right) & F_{y}<0,\end{cases}
\end{aligned}
$$

where $V_{1}, V_{2}, V_{3}$, and $V_{4}$ are the voltages measured from R1, R2, R3, and R4, respectively. $f_{1}, f_{2}, f_{3}$, and $f_{4}$ are the functions between voltage and the external force in different measuring phases, respectively.

The deflection of the core axis can be calculated as follows:

$$
T=f\left(F_{x}+F_{y}\right),
$$

where $f$ is the function of the pressure on the sensor and the deflection of the shaft under test.

The sensor involves several components, including circuit board, FSR layer, and elastic rubber layer. Since the FSR layer and the elastic rubber layer have some nonlinear characteristics, a proper calibration method is needed to determine the five functions (i.e., $f_{1}, f_{2}, f_{3}, f_{4}$, and $f$ ).

2.2. Prototype and Fabrication Processes. A prototype sensor is shown in Figure 3, and a slender cylindrical shaft (made by polyurethane) is used as the core shaft of the sensor. The length and diameter of the shaft are $140 \mathrm{~mm}$ and $15 \mathrm{~mm}$, respectively. The rubber layer, FSR film, and PCB plate are fixed at one terminal of the shaft between two fix rings, which are also made by polyurethane with the thickness (along the shaft direction) and diameter of $15 \mathrm{~mm}$ and $21 \mathrm{~mm}$, respectively. When the core shaft bends under external force, the stress on FSR will arise from the two fix rings, so the thickness of the two fix rings is one of the key elements relating to the sensitivities of the sensor. If the thickness is too small, the pressure on the FSR will be minor even though the adopted shaft is exactly the same.
TABLE 1: The typical characteristics of FSR material [13].

\begin{tabular}{lc}
\hline Item & Value \\
\hline Minimal actuation force & $\sim 0.2 \mathrm{~N}$ \\
Force sensitivity range & $\sim 0.2 \mathrm{~N}-20 \mathrm{~N}$ \\
Force repeatability single part & $\pm 2 \%$ \\
Hysteresis & $+10 \%$ Average \\
\hline
\end{tabular}

The FSR ring, the sensing cell in the sensor, is provided by the Interlink Electronics, USA [13]. FSR is a robust polymer thick film (PTF) device that exhibits a decrease in resistance with the increase of force exerted on the surface of the sensor. The force sensitivity of FSR is optimized for the use in human touch control of electronics devices, such as automotive electronics, medical systems, and industrial and robotics applications. The typical features of the FSR material used here are listed in Table 1, showing good sensitivity, stable repeatability, and large measurement range of the FSR material.

The silicone elastomer layer (rubber layer) is produced by a local manufacturer (Shenzhen Mroow Electronics Co., Ltd, China). The elastomer layer is adopted to transmit and filter forces. However, in practice the hardness of the elastomer has a strong influence on the performance of the sensor, especially the sensitivity and measurement range. The variable thickness and hardness of this elastomer layer make the theoretical estimation impossible. Therefore, the silicon elastomer with a shore hardness ranging from $10 \mathrm{~A}$ to $70 \mathrm{~A}$ has been tested on the effects of elastomer hardness. Compared with the other variable hardness test results, the hardness of $60 \mathrm{~A}$ was determined to be used in this work, and the thicknesses of the elastomer layers were fixed at $0.5 \mathrm{~mm}$.

In order to measure the bending deflection and direction, a ring-shaped electrode grid circuit with four detection areas has been designed in this paper. This is because the contact force of the sensor is distributed along the circumference of the shaft; the radial distribution modes of electrodes can improve the accuracy of detection results. As shown in Figure 4 , the red ring outside is the supply power, consisting of 72 electrodes, whereas a detection electrode is located between the two power electrodes. The detection electrodes are divided into four phases (indicated by different colors in Figure 4), but they are connected to each other by the inner ring in each phase. The four fan-shaped detection areas are placed symmetrically along both the $X$-axis and the $Y$-axis, with the ranges of central angles of $(-\pi / 4, \pi / 4]$, $(\pi / 4,3 \pi / 4],(3 \pi / 4,5 \pi / 4]$, and $(5 \pi / 4,7 \pi / 4]$, respectively. In order to effectively reduce the contact resistance and increase the oxidation stability, the metal electrodes with a height of $35 \mu \mathrm{m}$ were treated by the process of surface precipitation.

In order to ensure that the central angle of the FSR region bearing stress always keeps $90^{\circ}$ under the action of external force in any direction, the relationship between the inside radius $(r)$ of the circuit board and the outside radius $(R)$ of the fix ring should meet (3), as shown in Figure 5(a). The area bearing stress $(S)$ of FSR can be calculated by (4): 


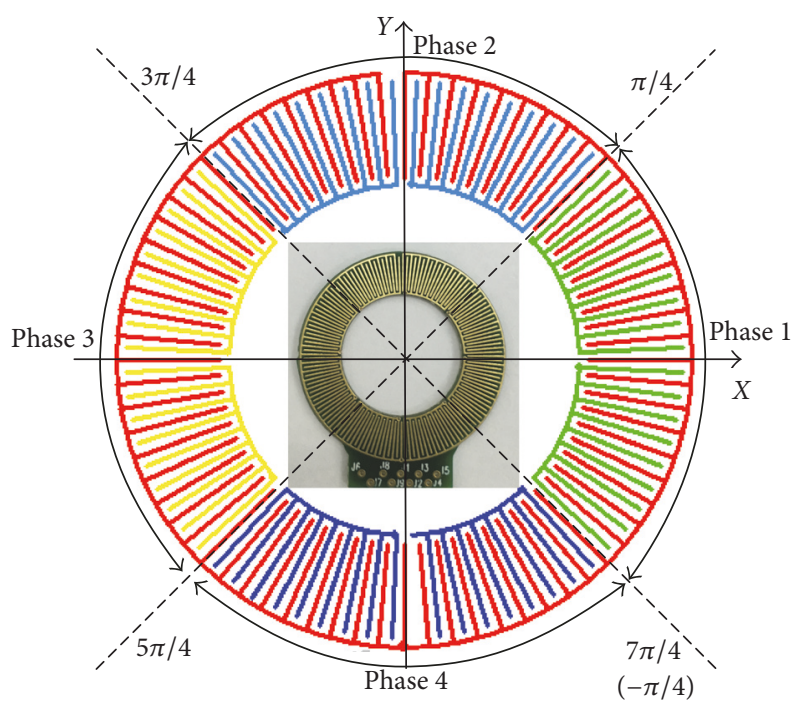

FIGURE 4: The circuit board and the distribution of the electrodes.

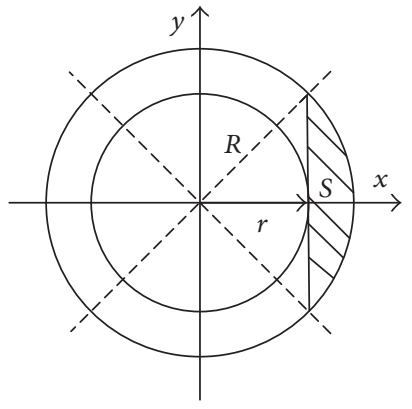

(a)

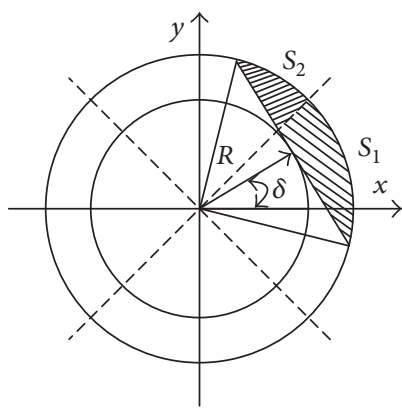

(b)
FIGURE 5: Schematic diagram of the FSR area under stress.

$$
\begin{aligned}
& r=R \cos \left(\frac{\pi}{4}\right) \\
& S=\frac{(\pi-2) R^{2}}{4} .
\end{aligned}
$$

As a result of the geometric constraints mentioned above, the FSR region under stress distributes only in one or two detection phases. The sum of the pressure measured in the two phases is equal to the total pressure acting on the sensor. Supposing that the angle between the direction of force acting on the sensor and the $+X$-axis is $\delta(\delta \in(0, \pi / 2]$, see Figure 5(b)), the stress area in the first and second phases (i.e., $S_{1}$ and $S_{2}$ ) can be computed by

$$
\begin{aligned}
& S_{1}=\frac{R^{2}[\pi-1-2 \delta-\tan (\pi / 4-\delta)]}{4} \\
& S_{2}=\frac{R^{2}[2 \delta-1+\tan (\pi / 4-\delta)]}{4} .
\end{aligned}
$$

Supposing that the normal stress distributes uniformly within the area of $S$, the value of resistance per unit area $\left(S_{0}\right)$

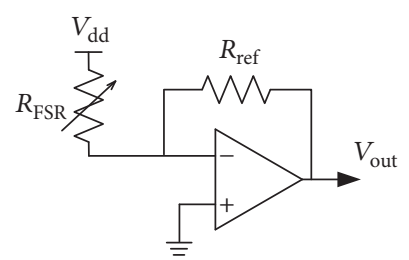

FIGURE 6: The simplified structure of measurement circuit for the FSR voltage.

is uniform, defined as $R_{0}$. The resistance of $S_{1}$ and $S_{2}$ can be obtained by

$$
\begin{aligned}
& R_{\mathrm{FSR} \_1}=\frac{R_{0} S_{0}}{S_{1}} \\
& R_{\mathrm{FSR} \_2}=\frac{R_{0} S_{0}}{S_{2}} .
\end{aligned}
$$

In order to get a voltage proportional to the area of FSR region under stress, an amperometric measurement circuit has been adopted, as shown in Figure 6. The output voltage $\left(V_{\text {out }}\right)$ of the sensor is shown in

$$
V_{\text {out }}=-\frac{R_{\mathrm{ref}} \cdot V_{\mathrm{dd}}}{R_{\mathrm{FSR}}},
$$

where $R_{\mathrm{FSR}}$ is the resistance of FSR under test and $V_{\mathrm{dd}}$ is the constant power supply. $R_{\text {ref }}$ can be used to adjust the sensitivity of the sensor. Thus, the output voltage of $S_{1}$ and $\mathrm{S}_{2}$ can be defined as follows:

$$
\begin{aligned}
& V_{\text {out_1 }}=-\frac{R_{\mathrm{ref}} \cdot V_{\mathrm{dd}}}{S_{0} \cdot R_{0}} \cdot S_{1} \\
& V_{\text {out_2 }}=-\frac{R_{\mathrm{ref}} \cdot V_{\mathrm{dd}}}{S_{0} \cdot R_{0}} \cdot S_{2} .
\end{aligned}
$$

The areas of $S_{1}$ and $S_{2}$ can be obtained through the measured voltages $V_{\text {out_1 } 1}$ and $V_{\text {out_2 }}$. Also, the bending direction can be calculated on the basis of $V_{\text {out_1 }}$ and $V_{\text {out_2 }}$.

$$
\delta=f_{5}\left(V_{\text {out }_{1}}, V_{\text {out }_{2}}\right)
$$

2.3. Scanning Method. In general, to get the entire shape of an elongated continuum robot, a number of deflection sensors will be needed. Thus, the number of lines for data collection should be cut down to minimize the integration circuit. Figure 7 presents the schematic diagram of a deflection sensor array, which is composed of six deflection sensors. If a constant current source or voltage source is used, the numbers of row lines and column lines to extract each component of force without interference are four and six, respectively. That is to say, a row line is connected to six force sensors simultaneously. A column line is connected to the power electrode of one deflection sensor. All of these lines should be connected to the processor, always at one end of the robot, which is responsible for $\mathrm{A} / \mathrm{D}$ conversion and data collection. 


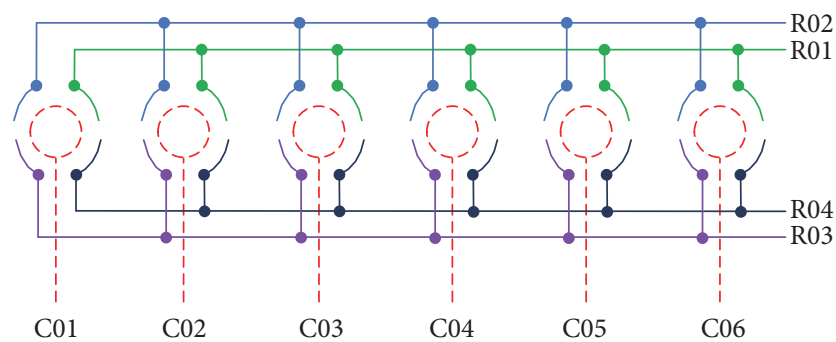

FIGURE 7: Schematic diagram of sensor array.

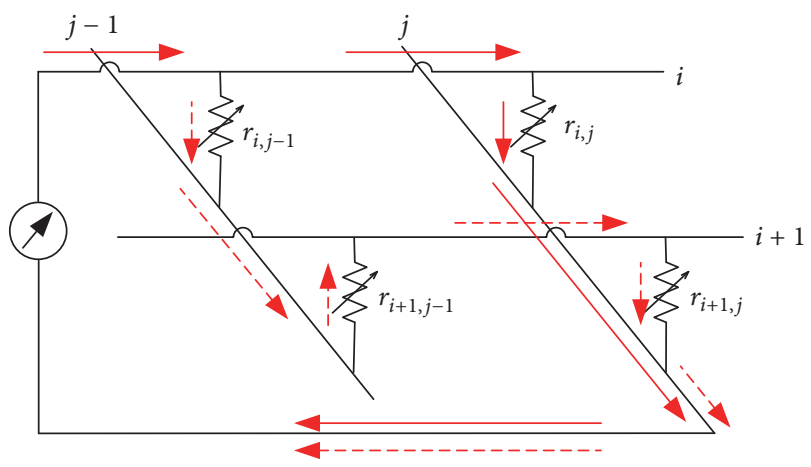

FIGURE 8: Schematic of crosstalk between adjacent elements [12].

Since an array of electrodes are used, the crosstalk between the adjacent deflection sensors becomes an important issue that needs to be considered. The basic principle of crosstalk is shown in Figure 8. In theory, when the scanning circuit scans the $i$-row electrode and the $j$-column electrode (the path of current is indicated by the solid arrows), only the resistance $r_{i, j}$ will be measured. However, the series pathway formed by $r_{i, j-1}, r_{i+1, j-1}$ and $r_{i+1, j}$ (indicated by the dashed lines with arrows) is also incorporated into the measurement loop, which will affect the measurement precision of the resistance $r_{i, j}$.

The variable $r_{v}$ is defined as the sum of $r_{i, j-1}, r_{i+1, j-1}$, and $r_{i+1, j}$; thus the actual resistance between the electrodes of $i$ and $j$ can be obtained through (11). If $r_{v} \gg r_{i, j}$, then $r_{i, j}^{m} \approx r_{i, j}$; else there will be a relatively large interference current through the measurement loop, which will bring bad affection on the accuracy of measurement [12].

$$
\begin{aligned}
& r_{v}=r_{i, j-1}+r_{i+1, j-1}+r_{i+1, j} \\
& r_{i, j}^{m}=r_{v} / / r_{i, j}=\frac{r_{v} \cdot r_{i, j}}{r_{v}+r_{i, j}} .
\end{aligned}
$$

In order to eliminate the effect of crosstalk, different methods have been proposed in the literatures, which can be broadly divided into two categories: the voltage feedback method [14] and the zero-potential method $[15,16]$. The basic mechanism of these two types of methods is to form an equal potential zone to eliminate the effect of crosstalk. However, the performance of zero-potential methods seems better than any circuit based on voltage feedback method, if the resistance values of the selected multiplexers and switches are

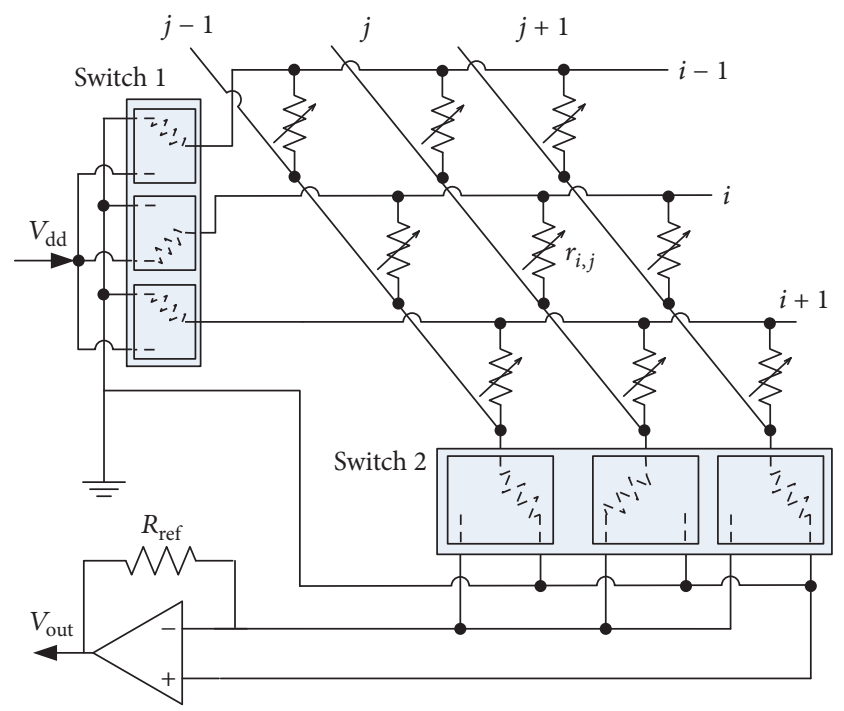

FIGURE 9: Simplified circuit based on the zero-potential method [12].

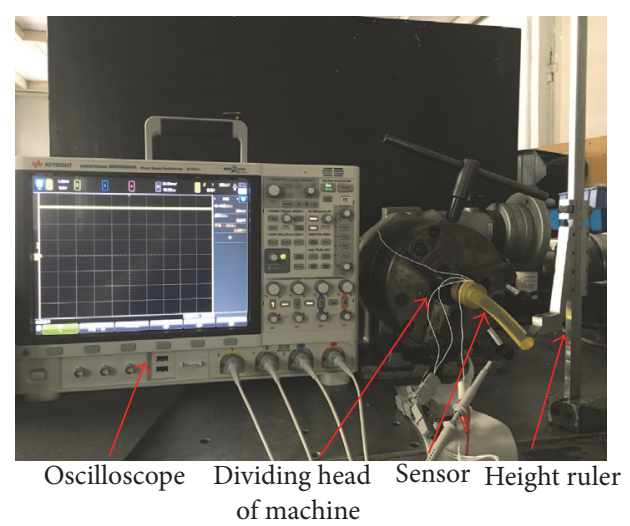

FiguRE 10: Experimental system for calibration.

proper [17]. Therefore, the zero-potential method has been adopted in this paper for the need of using an amperometric measure scheme. The simplified circuit based on the zeropotential method is shown in Figure 9. The resistance $r_{i, j}$ is placed into the measurement loop through the single pole double throw (SPDT) switches (i.e., switch 1 and switch 2). The driving voltage $V_{\mathrm{dd}}$ is applied on the current scanned driving electrodes (the $i$-row electrodes) by switch 1 , while the current scanned sampling electrodes (the $j$-column electrodes) are connected to the inversion input pin of the operational amplifier (op-amp) through switch 2. The two ends of the reference resistance $\left(R_{\text {ref }}\right)$, which also determines the sensitivity and measurement range of sensor, connect the inversion input pin and the output pin of the op-amp together. Furthermore, the noncurrent scanned electrodes are connected to the ground by two SPDT switches.

\section{Experimental Study}

3.1. Experimental System. To test the performance of the developed sensor, a calibration system has been built (Figure 10), which consists of a height ruler, a dividing head 


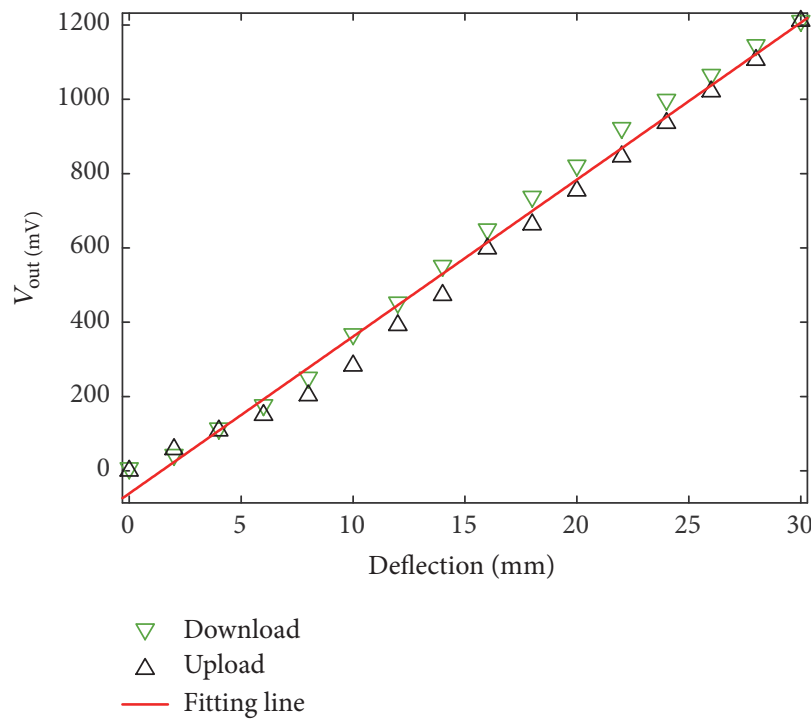

(a)

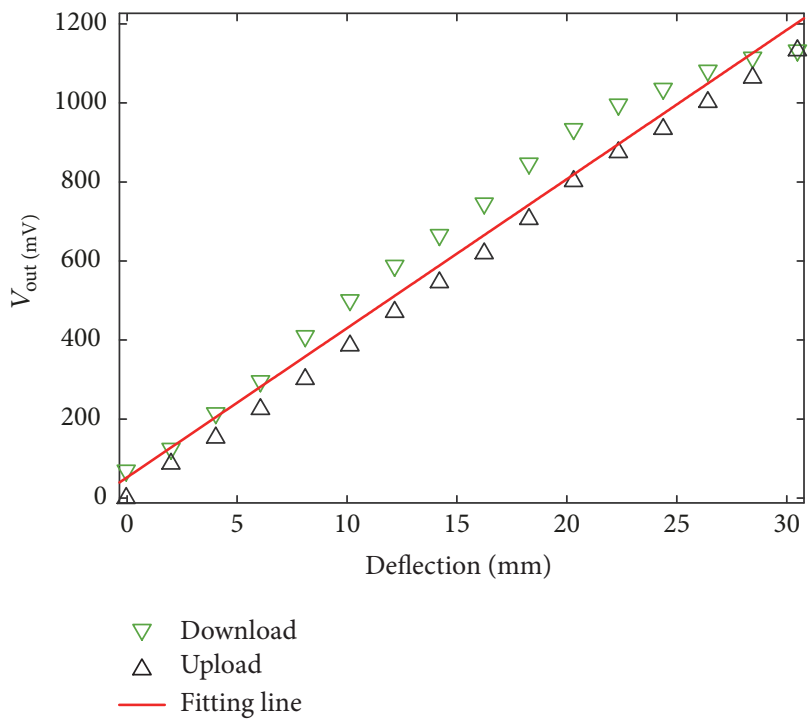

(c)

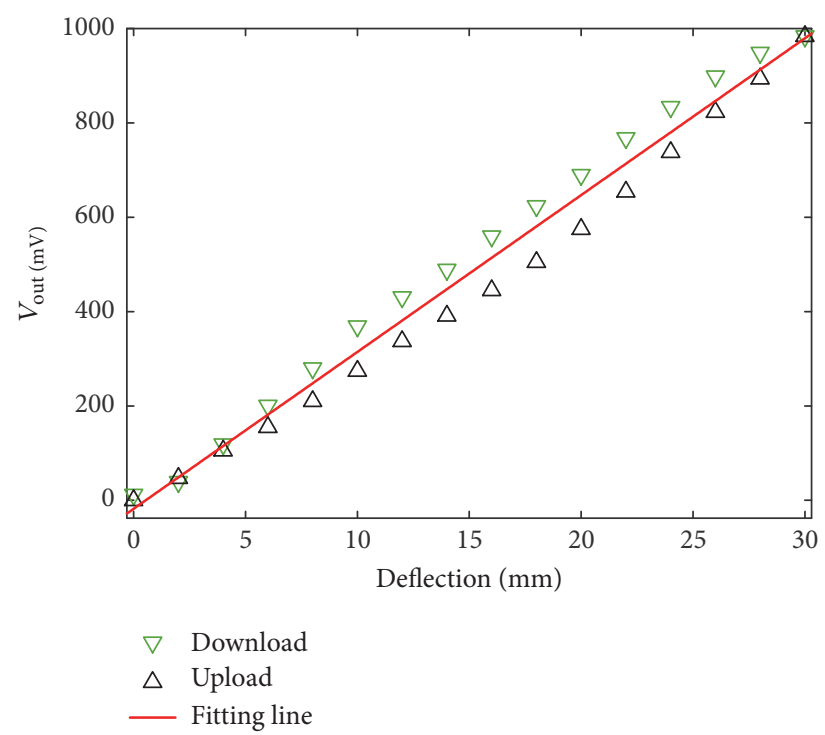

(b)

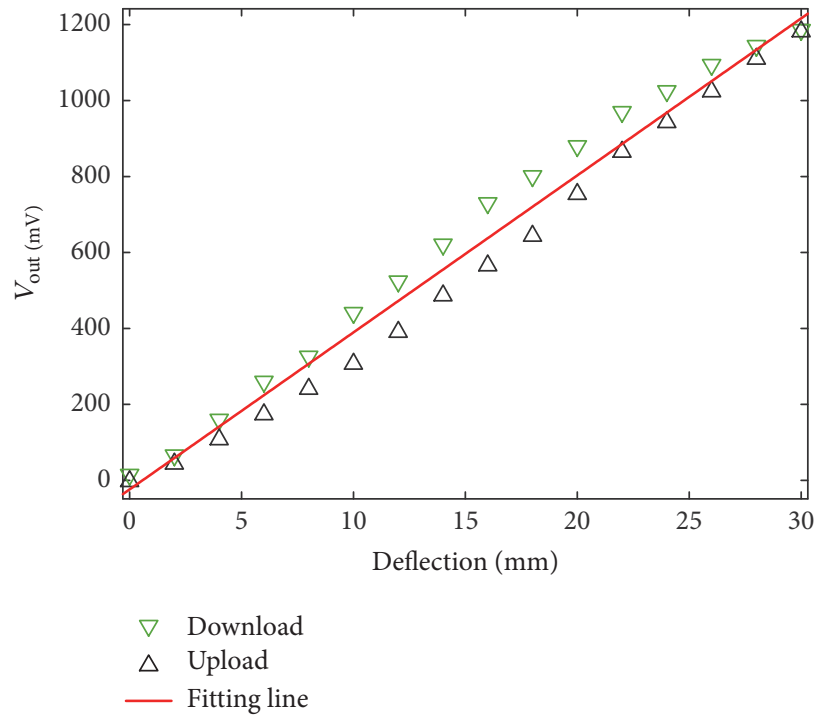

(d)

FIGURE 11: Input-output relationship for the force components in four directions. (a) $+x,(\mathrm{~b})+y,(\mathrm{c})-x$, and (d) $-y$.

of machine with three-jaw chuck, and an oscilloscope. The height ruler with the measurement accuracy of $0.02 \mathrm{~mm}$ is used to fix the suspending end of the shaft and measure its deflection. The dividing head is used to fix the base of sensor, helping to adjust the direction of the force imposed on the FSR easily with the resolution of $10^{\prime \prime}$. The oscilloscope is used to measure the output voltage of the sensor.

3.2. Bending Deflection Test. To obtain the relationship between the deflection of the sensor's suspending end and the output voltage, the calibration process is elaborated as follows:

(a) Measure the output voltage of the sensor without exerting any load.

(b) Fix the base of the sensor using the three-jaw chuck. (c) Adjust the angle of the chuck, letting the $+x$ (or $+y,-x,-y)$ direction of the sensor face vertically downward.

(d) Exert a force on the suspending end of the sensor by adjusting the height ruler.

(e) Record the deflection and the output voltage of the sensor at the same time.

During the experiments, the bending deflection at the suspending end of the core shaft increases from $0 \mathrm{~mm}$ to $30 \mathrm{~mm}$ first (regarded as upload) and then decreases back to $0 \mathrm{~mm}$ (regarded as download) by adjusting the height ruler with an interval of $2 \mathrm{~mm}$. The relationships between the deflection and the output voltage in the four directions are shown in Figure 11, where the red solid line is the fitting straight line averaged from the upload date and the download 
date with the same deflection. From the test data, it can be found that there is good linearity between the output voltages and the deflections in the four directions. The sensitivities of the prototype sensor are $40.37,32.8,37.77$, and $39.47 \mathrm{mV} / \mathrm{mm}$ for the $+x,+y,-x$, and $-y$ directions, respectively. The nonlinearities in the $+x,+y,-x$, and $-y$ directions are $5.4 \%$, $2.2 \%, 5.8 \%$, and $2.7 \%$, respectively. The hystereses of the sensor in the $+x,+y,-x$, and $-y$ directions are $7.1 \%, 12 \%$, $11.7 \%$, and $13.2 \%$, respectively. The sensitivity, nonlinearity, and hysteresis are defined as follows:

$$
\begin{aligned}
\text { Sensitivity } & =\frac{\max \left(V_{\text {out }}\right)}{\max (\text { Deflection })} \\
\text { Nonlinearity } & =\frac{\max \left(V_{\text {out }}-V_{F}\right)}{\max \left(V_{\text {out }}\right)} \\
\text { Hysteresis } & =\frac{\max \left(V_{\text {up }}-V_{\text {down }}\right)}{\max \left(V_{\text {out }}\right)} .
\end{aligned}
$$

3.3. Bending Orientation Test. The second experiment is designed to validate the feasibility of estimating the bending direction using the voltages from the two adjacent measuring phases. Taking phase 1 and phase 2 as an example, the experimental process is elaborated as follows:

(a) Adjust the dividing head and let the $+x$ direction of the sensor face vertically downward.

(b) Set the deflection of the core shaft to $10 \mathrm{~mm}$ by adjusting the height ruler.

(c) Turn the dividing head to the $+y$ direction and record the output voltages $\left(V_{\text {out-1 }}\right.$ and $\left.V_{\text {out-2 }}\right)$ of the two measuring phases simultaneously with interval of $5^{\circ}$.

As defined in Figure 5, the variation range of $\delta$ is $(0, \pi / 2]$. The test results are shown in Figure 12, where the red line indicates the relationship between $R_{S}$ (area ratio: $S 1 / S$ ) and $\delta$, whereas the blue line indicates the relationship between the measured value $R_{v}\left(V_{\text {out- } 1} /\left(V_{\text {out- } 1}+V_{\text {out- } 2}\right)\right)$ and $\delta$. The normal stress is fully imposed on phase 1 at the starting point, but the normal stress turns to be fully imposed on phase 2 when $\delta$ equals to $\pi / 2$. The experimental results show that $R_{v}$ seems almost consistent with the theoretical calculation value $R_{s}$. That is, the angle $\delta$ can be reliably obtained on the basis of the measured voltage from two adjacent detection phases.

\section{Discussion and Conclusions}

A new two-dimensional deflection sensor, taking FSR as its basic material, has been proposed and demonstrated in this work. The sensor consists of five concentric rings and is mounts vertically to the axis of the target shaft. When the bending of shaft occurs at the position where the sensor is mounted, the normal pressure will be imposed on the FSR film, thereby causing the change of FSR's resistance. A printed circuit board with electrode grids (divided into four measuring phases) and an integrated circuit with the ability of eliminating the crosstalk effect are designed to

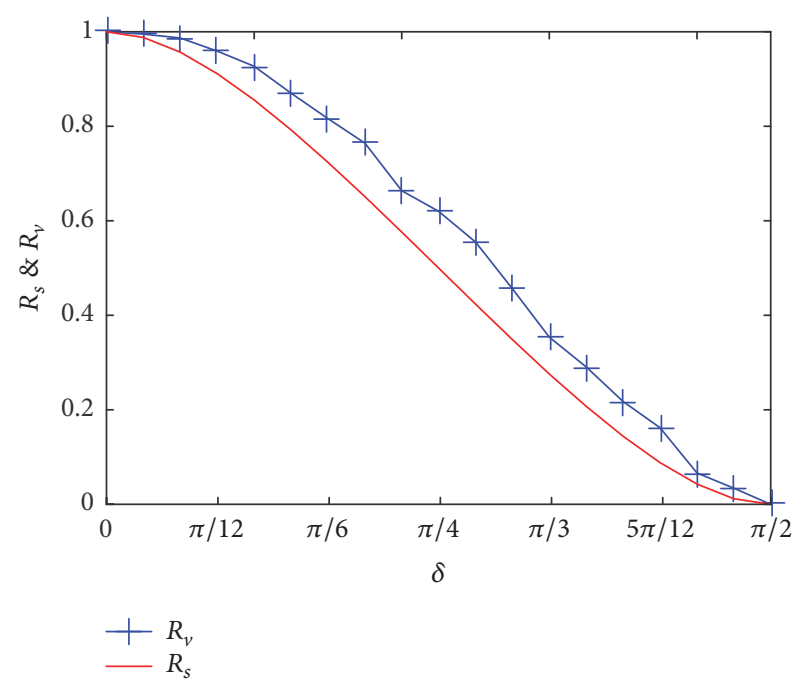

FIGURE 12: The relationship between the bending angle and the area ratio.

measure the output voltages of FRS film. Based on the specific calibration method, the deflection and the bending direction of the target shaft can be obtained from the measured voltages. The experimental results based on a prototype of the proposed deflection sensor show a good consistence between the theoretical value and the measured value. For a shaft with the length of $140 \mathrm{~mm}$, the measurement range of deflection can reach to $30 \mathrm{~mm}$. For the four measuring phases of the prototype sensor (in $+x,+y,-x$, and $-y$ directions), the sensitivities are $40.37,32.8,37.77$, and $39.47 \mathrm{mV} / \mathrm{mm}$, the nonlinearities are $5.4 \%, 2.2 \%, 5.8 \%$, and $2.7 \%$, and the hystereses are $7.1 \%, 12 \%, 11.7 \%$, and $13.2 \%$, respectively. Furthermore, the estimated bending direction is in good agreement with the actual direction. The proposed sensor can be used in many practical applications where the measurement of large and multidirection bending is required.

\section{Conflicts of Interest}

The authors declare that there are no conflicts of interest regarding the publication of this paper.

\section{Acknowledgments}

This work is supported in part by the National Natural Science Foundation of China (Grant no. 51505098), China Postdoctoral Science Foundation (Grant no. 2015M571403), Self-Planned Task (no. SKLRS201605C) of State Key Laboratory of Robotics and System (HIT), and the Foundation for Innovative Research Groups of the National Natural Science Foundation of China (no. 51521003).

\section{References}

[1] B. Kim, J. Ha, F. C. Park, and P. E. Dupont, "Optimizing curvature sensor placement for fast, accurate shape sensing of continuum robots," in Proceedings of the 2014 IEEE International 
Conference on Robotics and Automation, ICRA 2014, pp. 53745379, June 2014.

[2] S. C. Ryu and P. E. Dupont, "FBG-based shape sensing tubes for continuum robots," in Proceedings of the 2014 IEEE International Conference on Robotics and Automation, ICRA 2014, pp. 35313537, June 2014.

[3] H. Ren and P. E. Dupont, "Tubular enhanced geodesic active contours for continuum robot detection using 3D ultrasound," pp. 2907-2912.

[4] E. J. Lobaton, J. Fu, L. G. Torres, and R. Alterovitz, "Continuous shape estimation of continuum robots using X-ray images," in Proceedings of the 2013 IEEE International Conference on Robotics and Automation, ICRA 2013, pp. 725-732, May 2013.

[5] M. Mahvash and P. E. Dupont, "Stiffness control of surgical continuum manipulators," IEEE Transactions on Robotics, vol. 27, no. 2, pp. 334-345, 2011.

[6] A. Bajo and N. Simaan, "Kinematics-based detection and localization of contacts along multisegment continuum robots," IEEE Transactions on Robotics, vol. 28, no. 2, pp. 291-302, 2012.

[7] Y. Li, M. Miyasaka, M. Haghighipanah, L. Cheng, and B. Hannaford, "Dynamic modeling of cable driven elongated surgical instruments for sensorless grip force estimation," in Proceedings of the 2016 IEEE International Conference on Robotics and Automation, ICRA 2016, pp. 4128-4134, May 2016.

[8] Y. Li and B. Hannaford, "Gaussian process regression for sensorless grip force estimation of cable-driven elongated surgical instruments," IEEE Robotics and Automation Letters, vol. 2, no. 3, pp. 1312-1319, 2017.

[9] M. K. Dobrzynski, R. Pericet-Camara, and D. Floreano, "Contactless deflection sensor for soft robots," in Proceedings of the 2011 IEEE/RSJ International Conference on Intelligent Robots and Systems: Celebrating 50 Years of Robotics, IROS'11, pp. 1913-1918, September 2011.

[10] X. Meng, G. W. Roberts, A. H. Dodson, S. Ince, and S. Waugh, "GNSS for structural deformation and deflection monitoring : implementation and data analysis," in Proceedings of the $3 \mathrm{rd}$ IGA/12th FIG Symposium, pp. 22-24, May 2006.

[11] Y. Shapiro, A. Wolf, and G. Kósa, "Piezoelectric deflection sensor for a bi-bellows actuator," IEEE/ASME Transactions on Mechatronics, vol. 18, no. 3, pp. 1226-1230, 2013.

[12] T. Zhang, H. Liu, L. Jiang, S. Fan, and J. Yang, "Development of a flexible 3-D tactile sensor system for anthropomorphic artificial hand," IEEE Sensors Journal, vol. 13, no. 2, pp. 510-518, 2013.

[13] Datasheet_FSR, https://www.interlinkelectronics.com/datashee-tts/Datasheet_FSR.pdf.

[14] B. Tise, "A compact high resolution piezoresistive digital tactile sensor," in Proceedings of the IEEE International Conference on Robotics and Automation, pp. 760-764, Philadelphia, PA, USA, April 1988.

[15] W. D. Hillis, "A High-Resolution Imaging Touch Sensor," The International Journal of Robotics Research, vol. 1, no. 2, pp. 3344, 1982.

[16] M. Shimojo, A. Namiki, M. Ishikawa, R. Makino, and K. Mabuchi, "A tactile sensor sheet using pressure conductive rubber with electrical-wires stitched method," IEEE Sensors Journal, vol. 4, no. 5, pp. 589-596, 2004.

[17] H. Liu, Y. Zhang, Y. Liu, and M. Jin, "Measurement errors in the scanning of resistive sensor arrays," Sensors and Actuators A: Physical, vol. 10, pp. 1016-1023, 2010. 


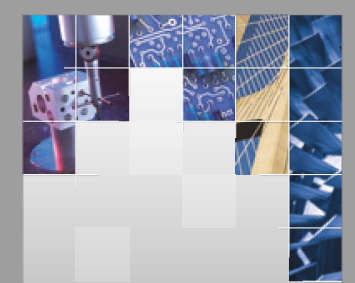

\section{Enfincering}
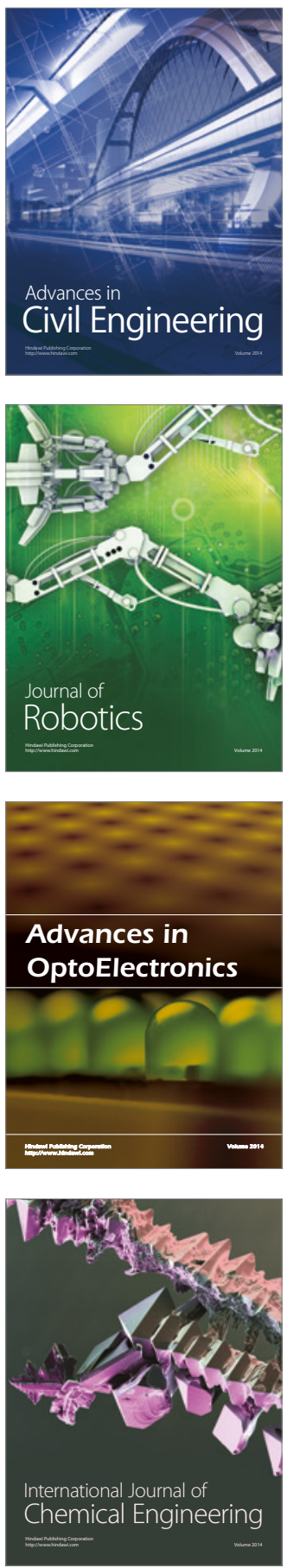

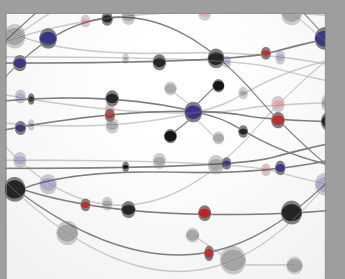

The Scientific World Journal

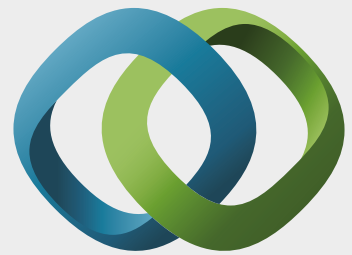

\section{Hindawi}

Submit your manuscripts at

https://www.hindawi.com
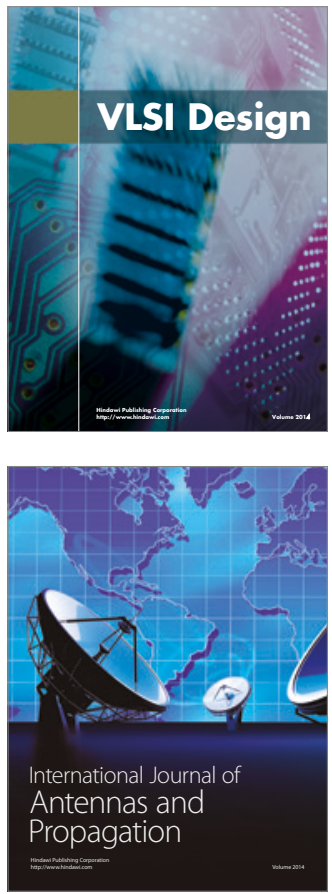

\section{Rotating}

Machinery
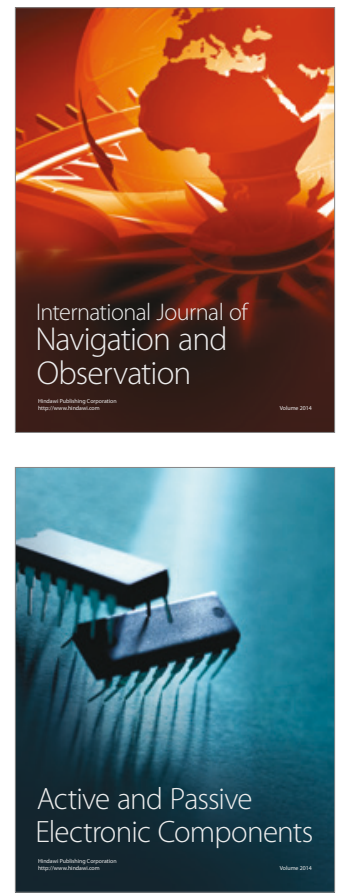
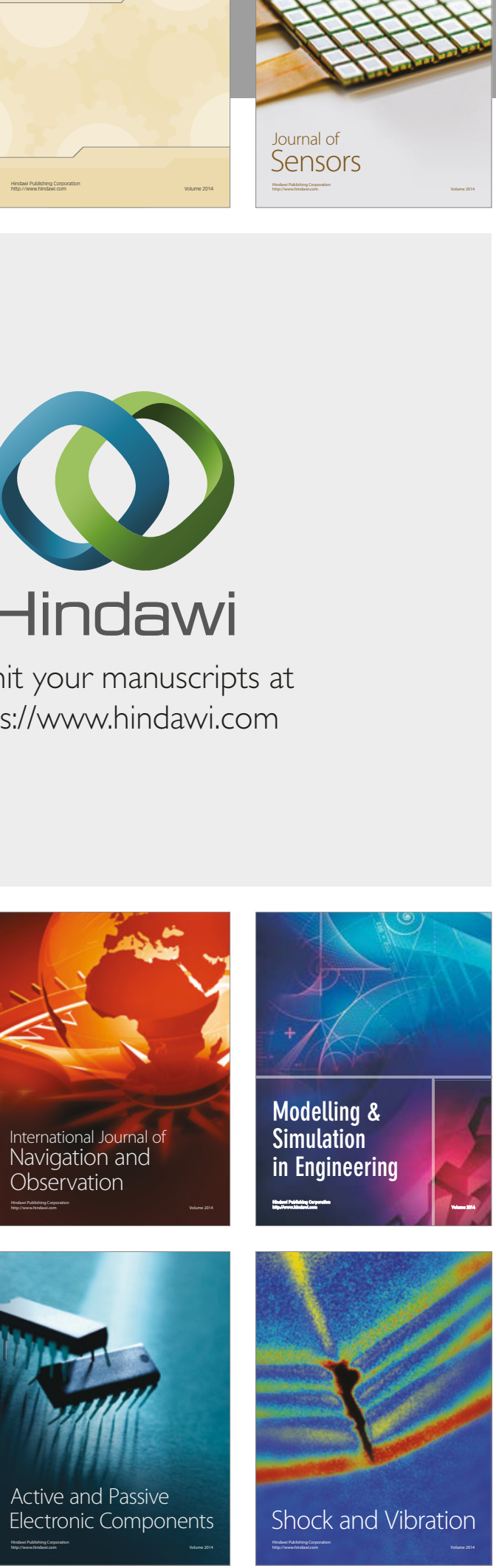
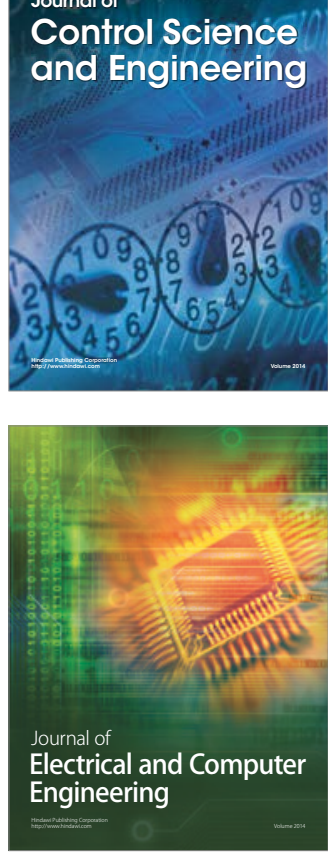

Distributed

Journal of

Control Science

and Engineering
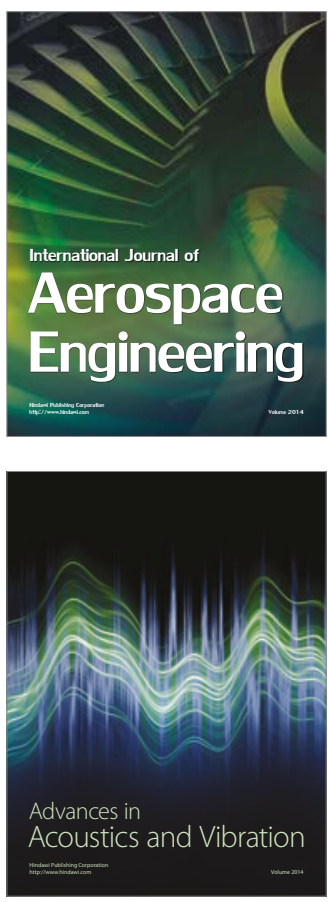

Sensor Networks 